\title{
An Accessible Keyboard for Android Devices as a Means for Promoting Braille Literacy
}

\author{
http://dx.doi.org/10.3991/ijim.v10i2.5527 \\ Paul D. Hatzigiannakoglou, Maria T. Kampouraki \\ University of Macedonia, Greece
}

\begin{abstract}
BrailleOne is a mobile application designed to provide blind and visually-impaired Braille readers with an accessible keyboard for manual text input on their Android smartphones or tablets. Its purpose is to make use of adolescents and young adults' excitement for technology in order to facilitate Braille learning and daily practice. The results of a pilot evaluation study suggest that BrailleOne could contribute to the overcoming of accessibility barriers in the field of text entry.
\end{abstract}

Index Terms-Braille, Android, keyboard, mobile application, accessibility, blindness, visual impairment.

\section{INTRODUCTION}

Braille is a tactile reading and writing code used by individuals with blindness or other forms of visual impairment. In Grade I Braille, most written characters can be represented by a "cell". Cells consist of 6 dots arranged in two columns of three dots each. Different combinations of $1-5$ dots in a cell are used to represent letters and other symbols.

The proportion of visually-impaired individuals who are proficient in Braille is gradually decreasing, even though blind or visually-impaired Braille readers are more likely to engage in higher education, to be employed and to enjoy a better quality of life $[1,2]$. One possible explanation is that digital techniques, such as screen readers, are becoming increasingly popular.

BrailleOne is a mobile learning application that aims to enable users to type text on a touch screen using Braille code. It serves a dual purpose: firstly, it is an accessible keyboard for sending messages and emails; and secondly, it is a means for efficient and enjoyable Braille learning and practice. BrailleOne could help blind and visuallyimpaired people benefit from the long-term effects of Braille literacy.

Part of this paper has been presented at the $1^{\text {st }}$ IMCL Student International Competition for Mobile Learning Apps, during the $9^{\text {th }}$ International Conference on Interactive Mobile Communication Technologies and Learning, which was held in Thessaloniki, Greece, November 19-20 2015.

\section{RELATED WORK}

Towards mobile keyboard accessibility, initial ideas include a gesture-driven software button [3] and an isometric joystick [4]. Other options used a mobile keypad to navigate through the visual alphabet or matching specific keys to the 6 Braille dots [5]. More recently, a multi-touch text input method was introduced [6] and Braille input approaches with one [7] or multiple fingers [8] were em- ployed. Another suggestion is that for Braille input purposes, each cell should be decomposed into 2-dot subsymbols [9]. The application in References [10, 11] requires users to hold their smartphones in an irregular position in order to produce Braille code on a touch screen. Finally, a Braille keyboard comparable to BrailleOne has recently been presented, but it runs on iOS devices only [12].

\section{DESIGN AND DEVELOPMENT PROCESS}

BrailleOne consists of one main scene (Fig. 1). Braille text input can be effected with one hand; it takes two moves to type a character. The middle and lower part of the screen is divided into three vertical sectors and is used as a one-hand keyboard. These three sectors represent Braille dots 1, 2 and 3 at first touch and secondly, dots 4, 5 and 6.

On BrailleOne, first column dots (i.e. 1, 2 and 3) are typed first and appear in blue. The remaining three dots (i.e. 4, 5 and 6) follow and appear in red. For example, " $\mathrm{m}$ " is represented by dots 1,3 and 4 in Braille code. To write "m", a user must first touch sectors 1 and 3 simultaneously (Fig. 2), and then touch sector 1 again for dot 4 (Fig. 3).

All letter characters, numbers and some punctuation marks are included in the current version of the application. Apart from Braille typing, the following functions are available: Delete character (swipe left), Leave space (swipe right), Repeat character (swipe down), Copy to clipboard (swipe up).

Voice output is used so that progress could be monitored at all times.

\section{QUALITATIVE RESULTS OF A PRELIMINARY EVALUATION}

After developing BrailleOne, we carried out a pilot usability test. We visited a school for blind students in Thessaloniki, Greece, and asked four young Braille readers to evaluate our application. Their feedback included ideas regarding swipe left/right functions and the need for more languages made available. All four interviewees agreed that an accessible Braille keyboard would be of value for blind and visually-impaired individuals who wish to use an Android smartphone or tablet. An 18-year-old blind boy, who had never used a smartphone before, managed to write his name on BrailleOne after a 10-min demonstration session. However, two of the interviewees admitted that they have purchased iOS devices and use a voice input method in order to avoid the existing accessibility barriers. 


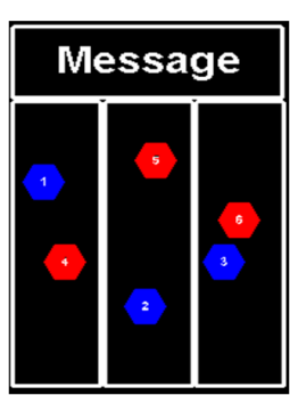

Figure 1. The layout of BrailleOne main scene.

\section{CONCLUSIONS AND FUTURE WORK}

BrailleOne is an accessible mobile keyboard for blind and visually-impaired individuals. It was designed to combine effective characteristics of previous applications and to overcome any further difficulties that have emerged.

Furthermore, BrailleOne is ideal for those who would rather avoid a standard mobile keyboard or voice input in noisy environments. Above all, adolescents and young adults with blindness or other forms of visual impairment are likely to benefit from this application, as it utilizes a popular technological device to practice and enhance their Braille typing skills. BrailleOne would be appealing to this latter age group, as it can eventually be used for writing text messages or keeping notes with no special equipment, no need for assistance and without attracting their peers' attention. In addition, this mobile learning application could facilitate and motivate Braille literacy and enable users with blindness or visual impairment improve their spelling skills. Concluding, the technological and educational features of BrailleOne are expected to be beneficial for people with such a disability.

There are further steps that should be taken before the potential of BrailleOne is fully used. A thorough usability investigation should be implemented. A training course on the use of BrailleOne would need to be developed and the average typing speed (words per minute) calculated for both blind and visually-impaired users with either proficient or elementary Braille knowledge, such that BrailleOne might be compared to other applications of its kind with regard to this specific aspect.

\section{REFERENCES}

[1] Rex, Evelyn J. "Issues related to literacy of legally blind learners." Journal of Visual Impairment and Blindness 83 (1989): 306-307, 310-312.

[2] Ryles, Ruby. "The impact of Braille reading skills on employment, income, education, and reading habits." Journal of Visual Impairment and Blindness 90 (1996): 219-226.

[3] Yfantidis, Georgios, and Evreinov, Grigori. "Adaptive blind interaction technique for touchscreens." Universal Access in the Information Society 4 (2006): 328-337, doi: 10.1007/s10209-0040109-7. http://dx.doi.org/10.1007/s10209-004-0109-7

[4] Wobbrock, Jacob O., Chau, Duen Horng and Myers, Brad A. "An alternative to push, press, and tap-tap-tap: Gesturing on an isometric joystick for mobile phone text entry." Paper presented at the SIGCHI Conference on Human Factors in Computing Systems, San Jose, CA, April 28-May 3, 2007.

[5] Guerreiro, Tiago, Lagoá, Paulo, Santana, Pedro, Gonçalves, Daniel and Jorge, Joaquim. "NavTap and BrailleTap: non-visual

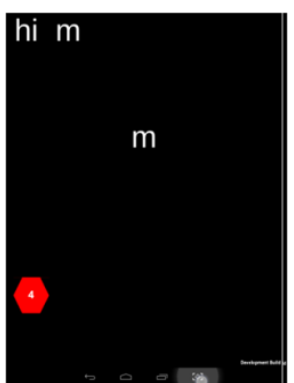

Figure 3. Second touch on sector 1 for " $\mathrm{m}$ ". ch on both sectors 1 and 3 for " $m$ ".

texting interfaces." Paper presented at the Rehabilitation Engineering and Assistive Technology Society of North America Conference (RESNA), Washington, D.C., 2008.

[6] Bonner, Matthew, Brudvik, Jeremy, Abowd, Gregory and Edwards, W. Keith. "No-look notes: accessible eyes-free multi-touch text entry." Paper presented at the $8^{\text {th }}$ International Conference on Pervasive Computing and Communications, Mannheim, Germany, March 29 - April 2, 2010.

[7] Oliveira, João, Guerreiro, Tiago, Nicolau Hugo, Jorge, Joaquim, and Gonçalves, Daniel. "BrailleType: Unleashing Braille over touch screen mobile phones." Paper presented at the $13^{\text {th }}$ IFIP TCI3 Conference on Human-Computer Interaction, Lisbon, Portugal, September 5-9, 2011.

[8] Azenkot, Shiri, Wobbrock, Jacob O., Prasain, Sanjana and Ladner, Richard, E. "Input finger detection for nonvisual touch screen text entry in Perkinput." Paper presented at the Graphics Interface, Toronto, ON, Canada, May 28-30, 2012.

[9] Mascetti, Sergio, Bernareggi, Cristian and Belotti, Matteo. "TypeInBraille: a Braille-based typing application for touchscreen devices." Paper presented at the $13^{\text {th }}$ International ACM SIGACCESS Conference on Computers and Accessibility, Dundee, Scotland, U.K., October 24-26, 2011.

[10] Frey, Brian, Southern, Caleb and Romero, Mario. "BrailleTouch: mobile texting for the visually impaired." Paper presented at the $6^{\text {th }}$ International Conference on Universal Access in HumanComputer Interaction, Orlando, FL, July 9-14, 2011.

[11] Southern, Caleb, Clawson, James, Frey, Brian, Abowd, Gregory D. and Romero, Mario. "An evaluation of BrailleTouch: mobile touchscreen text entry for the visually impaired." Paper presented at the $14^{\text {th }}$ International Conference on Human-Computer Interaction with Mobile Devices and Services, San Francisco, CA, September 21-24, 2012.

[12] Šepić, Barbara, Ghanem Abdurrahman, and Vogel Stephan. "BrailleEasy: one-handed Braille keyboard for smartphones." Paper presented at the $13^{\text {th }}$ AAATE Conference, Budapest, Hungary, 9-12 September, 2015.

\section{AUTHORS}

P. D. Hatzigiannakoglou is an electronic engineer, he holds a master degree in Information Systems (MIS) from the University of Macedonia, and he is a Ph.D. candidate at the same University. For several years he has designed and developed serious games applications (e-mail: pxatzi@uom.gr).

M. T. Kampouraki is a special educational needs (SEN) teacher and a graduate student of the Department of Educational and Social Policy at the University of Macedonia. She has been awarded a scholarship by the Christodoulakeio Foundation, House for the Kalymnian Female Student. (e-mail: kampourakimaria@gmail.com).

Submitted 28 January 2016. Published as resubmitted by the authors 24 March 2016. 